\title{
Effect of synbiotic yogurt fortified with monk fruit extract on hepatic lipid biomarkers and metabolism in rats with type 2 diabetes
}

\author{
Qingfeng Ban, ${ }^{1,2} \odot$ Xiaomeng Sun, ${ }^{1}$ × Yunqing Jiang, ${ }^{1}$ Jianjun Cheng, ${ }^{1 *}$ and Mingruo Guo ${ }^{3 *}$ (1) \\ ${ }^{1}$ College of Food Science, Northeast Agricultural University, Harbin, 150030, China \\ ${ }^{2}$ Key Laboratory of Dairy Science of Ministry of Education, Northeast Agricultural University, Harbin 150030, China \\ ${ }^{3}$ Department of Nutrition and Food Sciences, College of Agriculture and Life Sciences, University of Vermont, Burlington 05405
}

\begin{abstract}
Monk fruit extract (MFE) is widely used as a sweetener in foods. In this study, the effects of the consumption of MFE-sweetened synbiotic yogurt on the lipid biomarkers and metabolism in the livers of type 2 diabetic rats were evaluated. The results revealed that the MFE-sweetened synbiotic yogurt affected the phosphatidylcholines, phosphatidylethanolamines, phosphatidylglycerol, lysophosphatidic acids, lysophosphatidylcholines, lysophosphatidylethanolamines, lysophosphatidylglycerols, lysophosphatidylinositols, lysophosphatidylserines, and fatty acid-hydroxy fatty acids biomarkers in the livers of type 2 diabetic rats. In addition, the consumption of the MFE-sweetened synbiotic yogurt significantly altered 12 hepatic metabolites, which are involved in phenylalanine metabolism, sphingolipid metabolism, bile secretion, and glyoxylate and dicarboxylate metabolism in the liver. Furthermore, a multiomics (metabolomic and transcriptomic) association study revealed that there was a significant correlation between the MFE-sweetened synbiotic yogurt and the metabolites and genes involved in fatty acid biosynthesis, bile secretion, and glyoxylate and dicarboxylate metabolism. The findings of this study will provide new insights on exploring the function of sweeteners for improving type 2 diabetes mellitus liver lipid biomarkers.
\end{abstract}

Key words: type 2 diabetes mellitus, transcriptomic and metabolomic profile, synbiotic yogurt, monk fruit extract, lipid biomarker

\section{INTRODUCTION}

According to the statistics by the International Diabetes Federation, an estimated number of 463 million people suffer from diabetes globally, and this number is expected to increase to 700 million by 2045 . Type 2

Received August 25, 2021.

Accepted January 14, 2022.

*Corresponding authors: mguo@uvm.edu and jjcheng@neau.edu.cn diabetes mellitus (T2DM) accounts for over $90 \%$ of diabetes cases globally (International Diabetes Federation, 2019). Type 2 diabetes mellitus is a chronic metabolic disorder, which negatively affects the functions of organs (Uuh-Narváez et al., 2021). In addition, various metabolic disorders are observed at different stages of T2DM from the early to the later progression stages. Small-molecule metabolites have been identified and improved for the development of new strategies to prevent T2DM (Carrizo et al., 2017; Đurašević et al., 2020). Metabolomic investigation may provide a new perspective on the functional food treatment of T2DM.

Metabolomic analysis has attracted widespread research attention as an effective approach for investigating the relationship between metabolites and physiological pathology (Zhao et al., 2018). As a type of metabolic disorder, T2DM is characterized by unusual carbohydrates, lipids, and protein metabolism. As primary bioindicators, AA, lipids, and peptides can be used to provide biological information and assistance to properly understand diseases (Zhang et al., 2012). Therefore, it is essential to investigate the role of phosphatidyl metabolites in the pathogenesis of diabetes during diabetes management. Metabolomic experiments can be conducted in the following 2 ways: untargeted and targeted methods. Untargeted metabolomic analysis is effective for identifying putative biomarkers, as well as verifying experimental results (Sas et al., 2015). This indicates that metabolomic analysis is an effective tool for assessing the metabolic profiles of diabetes and therapeutic effects (Lu et al., 2013; Sas et al., 2015).

Previous studies have reported the promising potential of functional foods for the prevention and treatment of T2DM diseases (Carter et al., 2010; Watson and Preedy, 2019). A synbiotic yogurt is a yogurt containing prebiotics and probiotics (Schrezenmeir and de Vrese, 2001). Previous studies have reported that the consumption of synbiotic yogurt is beneficial for the treatment of T2DM (Pandey et al., 2015; Ban et al., 2020a). However, a high-sugar content is added to lowsugar fermented dairy products, particularly yogurts, to improve their taste (Gijsbers et al., 2016; Wan et al., 
2021). Oral administration of excessive sugar has been identified as one of the major factors that increases susceptibility to T2DM (Howard and Wylierosett, 2002). Therefore, tremendous efforts have been devoted to develop effective strategies for reducing the sugar content in dairy products, such as the replacement of sugar with low- or noncaloric alternative sweeteners (Howard and Wylierosett, 2002; Murphy and Johnson, 2003).

Siraitia grosvenorii is commonly known as monk fruit (Luo han guo). Monk fruit extract (MFE) has attracted attention as a promising alternative to sugar owing to its high sweetness level, which is 300 times higher than that of sucrose (Fang et al., 2017). In addition, previous studies have demonstrated the liver protection, antihyperglycemic, and anti-inflammatory properties of monk fruit (Li et al., 2014). Accordingly, MFE has been used as a nonnutritive sweetener in the United Stated, Japan, and China (Li et al., 2014). Additionally, MFE has attracted increasing global attention in food and dairy beverage industries, owing to the increasing demand for nonnutritive sweeteners (Pawar et al., 2013). Particularly, MFE has been used as a nonnutritive sweetener alternative in low-calorie foods for diabetes patients. Both MFE and mogrosides are beneficial for diabetes patients in that they enhance the rate of blood glucose uptake (Harada et al., 2016; Liu et al., 2019). Accordingly, numerous studies have extensively investigated the effects of nonnutritive sweeteners on human or rats using metabolomic analysis (Vamanu et al., 2019).

However, despite the numerous studies on products fortified with nonnutritive sweetener, studies on their effects on the liver of T2DM patients are rare. Moreover, it is essential to investigate the effects of the oral administration of nonnutritive sweetener products on the liver. It is essential to understand the biological mechanisms of complex disease using "omic" technologies (transcriptomics, proteomics, and metabolomics), which have facilitated the investigation of protein, lipid, and peptide. From a dietary intervention perspective, nonnutritive sweeteners are dietary signals that can be detected by cellular sensor systems to influence gene and protein expression, thus enhancing the production of metabolites. Therefore, in this study, we combined transcriptomic and metabolomic approaches to evaluate the effects of synbiotic yogurt sweetened with different sweeteners on lipid biomarkers and metabolism in liver tissues using high-fat diet and streptozotocin (STZ)-induced T2DM rats.

\section{MATERIALS AND METHODS}

\section{Materials}

The MFE powder was purchased from Huacheng Biomedical Company. The composition of MFE based on the product specification was as follows: $6.81 \%$ moisture, $3.45 \%$ total phenolics, $2.24 \%$ total flavonoids, and $87.5 \%$ total mogrosides. The STZ was purchased from Sigma-Aldrich. Whole milk was purchased from Mengniu Dairy, and pelletized high-fat diet (45\% fat) was purchased from Jiangsu Medicine Biomedical Co., Ltd. The composition of the normal diet and high-fat diet is shown in Supplemental Table S1 (https://data .mendeley.com/datasets/6r9xwsbhx7/1, Ban, 2022). The ABY-8 (Chr. Hansen), which was a blend of Streptococcus thermophilus and Lactobacillus delbrueckii ssp. bulgaricus, with Bifidobacterium BB-12 and Lactobacillus acidophilus LA, was used for the starter adjuncts. The $93 \%$ inulin was purchased from Zhangye Biomedical Co., Ltd. All other chemicals used in this study were of analytical grade.

\section{Preparation of the Synbiotic Yogurt}

Synbiotic yogurt was prepared based on a method reported in a previous study of Ban et al. (2020b). First, the whole milk was heated to $55^{\circ} \mathrm{C}$, after which sucrose $(30 \mathrm{mg} / \mathrm{mL})$ or MFE $(30 \mathrm{mg} / \mathrm{mL})$, and inulin $(10 \mathrm{mg} /$ $\mathrm{mL}$ ) were added. Subsequently, the mixture was heated at $80^{\circ} \mathrm{C}$ for $15 \mathrm{~min}$, after which the mixture was cooled rapidly to $43^{\circ} \mathrm{C}$. Thereafter, $0.3 \mathrm{mg} / \mathrm{mL}$ of ABY-8 was added to the mixture, and the mixture was fermented at $43^{\circ} \mathrm{C}$ for $5 \mathrm{~h}$ to obtain the synbiotic yogurt. The yogurt was stored at $4^{\circ} \mathrm{C}$ for further use. The average composition of the yogurt samples is shown in Supplemental Table S2 (https://data.mendeley.com/datasets/ 6r9xwsbhx7/1, Ban, 2022).

\section{Animal Models}

All experiments were conducted in accordance with the "Guidelines for Animal experimentation" established by the Laboratory Animal Care and Use Committee of Jiangnan University [SYXK (SU) 2016-0045. Approval number: JN. No20180915W0801120(194)].

Seven-week-old male Wistar rats (150-180 g, specificpathogen-free animals) were purchased from Slake Animal Centre. The rats were housed under the following conditions: $22 \pm 2^{\circ} \mathrm{C}$ temperature, $55 \pm 5 \%$ relatively humidity, and $12 \mathrm{~h}$ light-dark cycle. Ten rats were fed a normal diet (control group; CK), and 30 rats were fed a high-fat diet based on their BW. The rats fed the high-fat diet were divided into 3 groups as follows: diabetes control group (SIDR), control yogurt group (CY), and MFE-containing yogurt group (MYH). On the first day of the sixth week, all the rats were fasted for $12 \mathrm{~h}$, after which STZ $(30 \mathrm{mg} / \mathrm{kg}$ of BW) was injected into the intraperitoneal of the rats fed a high-fat diet to induce T2DM rats, and those fed on a basal diet 
were injected with an equivalent volume of $50 \mathrm{mmol} / \mathrm{L}$ citrate buffer ( $\mathrm{pH} 4.5$ ). From wk 2 to wk 7 , the SIDR group was injected with $30 \mathrm{mg} / \mathrm{mL}$ sucrose solution using a gavage method. The CY group received sucrose by orally feeding on $30 \mathrm{mg} / \mathrm{mL}$ control yogurt, and the MYH group received MFE orally by feeding them with $30 \mathrm{mg} / \mathrm{mL}$ synbiotic yogurt using a gavage method. On the basis of our previous study, the fasting glucose, oral glucose tolerance test, insulin, and homeostatic model assessment for insulin resistance results were reported in Ban et al. (2020a).

\section{Transcriptomic and Metabolomic Profiling of Liver Tissue Extracts}

The transcriptomic method reported by $\mathrm{Su}$ et al. (2020) was modified and used for the experiments. For this analysis, hepatic total RNA (50 mg of liver tissue) was extracted from the CK, SIDR, CY, and MYH rats ( $\mathrm{n}=3$ per group). The metabolomic method adopted in this study was based on the method reported by Meierhofer et al. (2014). For this analysis, $100 \mathrm{mg}$ of liver tissue was prepared from the CK, SIDR, CY, MYH rats ( $\mathrm{n}=6$ per group). Subsequently, liquid chromatography (LC) with tandem mass spectrometry (LC-MS/ MS) analyses were performed using a Vanquish UHPLC system (Thermo Fisher) coupled with an Orbitrap Q Exactive series mass spectrometer (Thermo Fisher).

The peak alignment, peak picking, and quantitation for each metabolite were performed by processing the raw metabolomic data files generated by LC-MS/MS using the Compound Discoverer 3.1 (CD3.1, Thermo Fisher). The main processing parameters were set as follows: retention time tolerance, $0.2 \mathrm{~min}$; actual mass tolerance, $5 \mathrm{ppm}$; signal intensity tolerance, 30\%; signal-to-noise ratio, 3; and minimum intensity, 100,000.

\section{Statistical Analysis}

Statistical analyses were performed using the statistical software R (R version 3.4.3, https://www.r-project .org/), Python (Python version 2.7.6), and CentOS (CentOS release 6.6). When data were not normally distributed, normal transformations were attempted using the area normalization method. The metabolites were annotated using the Kyoto Encyclopedia Genomes (KEGG) database (http://www.genome.jp/kegg/). Principal components analysis (PCA) and partial least squares discriminant analysis (PLS-DA) were performed using metaX (a flexible and comprehensive software for processing metabolomics data). The significance $(P$-value) of the results was calculated using univariate analysis ( $t$-test) and ANOVA test. The me- tabolites with variable importance projection (VIP) $>1$ and $P$-value $<0.05$ were considered to be differential metabolites. In addition, the metabolites of interest were filtered using volcano plots, which were calculated based on the $\log _{2}$ (fold change) and $-\log _{10}(P$-value $)$ values of the metabolites. The functions of the metabolites and metabolic pathways were investigated using the KEGG database. The metabolic pathway enrichment of differential metabolites was performed, and when the ratios were satisfied by $\mathrm{x} / \mathrm{n}>\mathrm{y} / \mathrm{N}$, the metabolic pathway was considered to be enriched. When the $P$-value of the metabolic pathway was $<0.05$, the enrichment of the metabolic pathway was considered to be significant.

Subsequently, the transcriptomic and metabolomic data sets were integrated using a sparse partial least squares regression method, which was performed using the MixOmics package. Partial least squares is a supervised method that selects correlated variables (genes, metabolites) across the same samples by maximizing the covariance between the data sets. In this study, sparse partial least squares was used to select the most correlated variables using LASSO (least absolute shrinkage and selection operator) penalization on the pair of loading vectors, as described by Mesnage et al. (2018).

\section{RESULTS}

\section{Principal Component Analysis}

The PCA analysis enabled a close and careful observation of the overall distribution trend of the samples. In addition, this analysis enabled the identification of possible discrete points. The positive- and negative-ion modes of the PCA score plots are shown in Supplemental Figure S1 (https://data.mendeley.com/datasets/ 6r9xwsbhx7/1, Ban, 2022). The 8-wk-long MYH gavage enabled the distinction of the MYH group from the SIDR group, owing to the therapeutic effect of the MFE-sweetened synbiotic yogurt. In addition, MFE was more effective for the treatment of diabetes than sucrose; thus, MFE was used as the main bioactive ingredients in the MYH group. The percentage of the explained variance in the positive-ion mode for principal components 1 and 2 were 31.03 and $10.53 \%$, respectively (Supplemental Figure S1A), and those in the negative-ion mode were 27.43 and $19.43 \%$, respectively (Supplemental Figure S1B).

\section{Identification of Potential Biomarkers}

The liver metabolites data from the CK (data not shown), CY, MYH, and SIDR rats obtained using LC- 
MS/MS were demonstrated in the PLS-DA score plot (Supplemental Figure S2A-H, https://data.mendeley .com/datasets/6r9xwsbhx7/1, Ban, 2022). The PLSDA score plots of the CY and SIDR groups were notably different (Supplemental Figure S2A and B). The model demonstrated a high predictability for $R^{2} Y$ and $Q^{2} Y$ values. The positive and negative $R^{2} Y$ and $Q^{2} Y$ parameters predicted by the model were 0.98 and 0.47 , and 0.94 and 0.56 , respectively. In addition, the $R^{2} Y$ and $\mathrm{Q}^{2} \mathrm{Y}$ parameters required by the software for the classification of the MYH and SIDR groups were 0.99 and 0.91 in positive mode, and 0.99 and 0.91 in negative mode, respectively, which were stable for further prediction on the MYH versus SIDR groups (Supplemental Figure S2C and D). The contribution of the variables between CY and SIDR, as well as those between MYH and SIDR, were investigated, and the plots of these values are shown in Supplemental Figure S2E to H. The plots suggested that the models were not overfitted. In addition, the results verified the successful development of T2DM diabetes in the rats via high-fat diet and STZ injection. In addition, the score plots of the CY- and MYH-treated rats were completely different from that of the SIDR-treated rats, owing to the effect of the yogurt feeding.

The results revealed that 555 metabolites were detected in the positive mode and 390 metabolites were detected in the negative mode (data not shown). In addition, CY treatment significantly affected 41 metabolites in the positive mode, whereas 58 metabolites were affected in the negative mode $(P<0.05)$. Further, MYH treatment significantly altered 102 metabolites in the positive mode, whereas 95 metabolites were affected in the negative mode $(P<0.05)$. To demonstrate the relationship between the 4 groups, a Venn diagram was constructed. The Venn diagram revealed that 17 significant metabolites in the positive mode were detected in both the CY and SIDR groups, and 29 significant metabolites in the negative mode were detected in both groups. In addition, 49 significant metabolites in the positive mode and 44 significant metabolites in the negative mode were detected in both the MYH and SIDR groups (Supplemental Figure S3, https://ata .mendeley.com/datasets/6r9xwsbhx7/1, Ban, 2022).

\section{Analysis of the Phospholipids Biomarkers in the Livers}

A VIP value of $>1$ and $P<0.05$ of the $t$-test of phospholipids biomarkers were considered significantly different. In the liver of the rats, 27 phospholipids biomarkers, including 6 lysophosphatidic acids (lysoPA), 5 lysophosphatidylcholines (lysoPC), 4 lysophosphatidylethanolamines (lysoPE), 1 lysophosphatidylglycerol (lysoPG), 1 lysophosphatidylinositol (lysoPI), 1 lysophosphatidylserines (lysoPS), 2 phosphatidylcholines (PC), 4 phosphatidylethanolamines $(\mathbf{P E})$, and 3 phosphatidylglycerols (PG) were significantly downregulated in the CY and SIDR groups $(P<$ $0.05)$. In addition, only 2 phospholipid biomarkers [PC (19:0/20:5), (20:2/20:3)] were significantly upregulated $(P<0.05)$ in the groups (Table 1$)$. Further, 27 phospholipids biomarkers, including 2 lysoPA, 2 lysoPC, 3 lysoPE, 2 lysoPG, 3 PC, 4 PE, 1 PI, and 10 PG, were significantly downregulated in the liver of the $\mathrm{MYH}$ and SIDR groups $(P<0.05)$. Additionally, 16 phospholipids biomarkers, including 1 lysoPA, 3 lysoPC, 3 lysoPE, 2 lysoPI, 4 lysoPS, and 3 PC, were upregulated in all the groups (Table 2).

\section{Analysis of the Fatty Acid-Hydroxy Fatty Acids Biomarkers in the Livers}

A VIP value of $>1$ and $P$-value $<0.05$ of the $t$-test of the fatty acid-hydroxy fatty acids (FAHFA) biomarkers was considered significantly different. Fourteen FAHFA biomarkers, including FAHFA (20:5/22:3), FAHFA (22:6/22:3), FAHFA $(22: 5 / 20: 2)$, FAHFA (18:3/22:3), FAHFA (20:4/18:1), FAHFA (18:2/18:0), FAHFA (22:6/22:5), FAHFA (22:6/20:3), FAHFA (18:2/18:1), FAHFA (22:5/22:4), FAHFA (20:4/22:4), FAHFA (14:1/22:3), FAHFA (16:2/22:3), and FAHFA $(20: 5 / 21: 1)$, were significantly upregulated in the livers of the CY and SIDR groups $(P<0.05$; Table 3$)$. In addition, 5 FAHFA biomarkers, including FAHFA (2:0/26:4), FAHFA (14:1/22:3), FAHFA (16:0/2:0), FAHFA (20:5/22:3), and FAHFA (16:1/18:3), were significantly upregulated in livers of the MYH and SIDR groups $(P<0.05)$. Further, only 2 FAHFA biomarkers, including FAHFA (20:1/22:3) and FAHFA (22:4/21:1), were upregulated in the livers of all the groups (Table 4). The FAHFA exhibited a good effect on the antidiabetic and anti-inflammatory properties of the livers. The abundance of FAHFA in the livers of the samples corresponded to the development of T2DM, indicating the significant influence of FAHFA on diabetes.

\section{Metabolic Pathway Analysis}

The MYH treatment significantly improved the metabolites, and the results are shown in Table 5. Using the aforementioned methods, a total of 12 potential biomarkers were identified in the groups. The identified biomarkers are involved in sphingolipid metabolism, glyoxylate and dicarboxylate metabolism, fatty acid biosynthesis, bile secretion, and phenylalanine me- 
tabolism in the liver. Compared with the SIDR group, the levels of N-acetylsphingosine, L-serine, D-serine, palmitoleic acid, taurolithocholic acid 3-sulfate, transcinnamic acid, and hydrocinnamic acid in the hepatic sample of the MYH group were higher. Furthermore, MYH treatment significantly decreased the levels of glycoursodeoxycholic acid, D-sphingosine, dehydrocholic acid, palmitoyl carnitine, and glycocholic acid in the hepatic sample $(P<0.05)$. Additionally, the VIP values of the level of N-acetylsphingosine, D-sphingosine, palmitoyl carnitine, and hydrocinnamic acid in the $\mathrm{MYH}$ and SIDR groups were above 1.8 (Table 3). Based on the KEGG pathway, a detailed pathway analysis was conducted to investigate the possible relationship between the metabolic pathways in the T2DM rats treated with MFE-sweetened synbiotic yogurt (Figure 1). The multiomics (metabolomic and transcriptomic) investigation revealed that long-term MYH feeding significantly affected multidimensional alterations in the livers of the rats. Particularly, long-term MYH feeding regulated fatty acid metabolism, bile secretion, and glyoxylate and dicarboxylate metabolism (Figure 2-3), and their related genes.

\section{DISCUSSION}

Ninety percent of diabetes patients suffer from T2DM annually. Therefore, it is imperative to develop advanced strategies for preventing T2DM. Products rich in MFE have been officially approved as functional additive foods in developed countries, such as Japan, Austria, and the United States. Monk fruit extract has attracted attention because of its antihyperglycemic, liver protection, anti-inflammatory, and sweetening properties $(\mathrm{Li}$ et al., 2014). Previous studies have focused on the effect of the oral administration of mogroside $\mathrm{V}$ on diabetic mice (Liu et al., 2019). In this study, we investigated the underlying mechanism involved in the metabolism regulation by MFE-sweetened synbiotic yogurt. Monk fruit extract has been recognized as a new nonnutritive functional additive, and the antidiabetes effect of MFE-sweetened synbiotic yogurt could be attributed to the presence of MFE, as well as the synergistic effect of both prebiotics and probiotics. The results of this revealed that MFE-sweetened synbiotic yogurt may be a good dairy product for T2DM patients. The investigation of the change in the hepatic transcriptomic and

Table 1. Identified phospholipids biomarkers in the diabetic rats in the control yogurt (CY) group

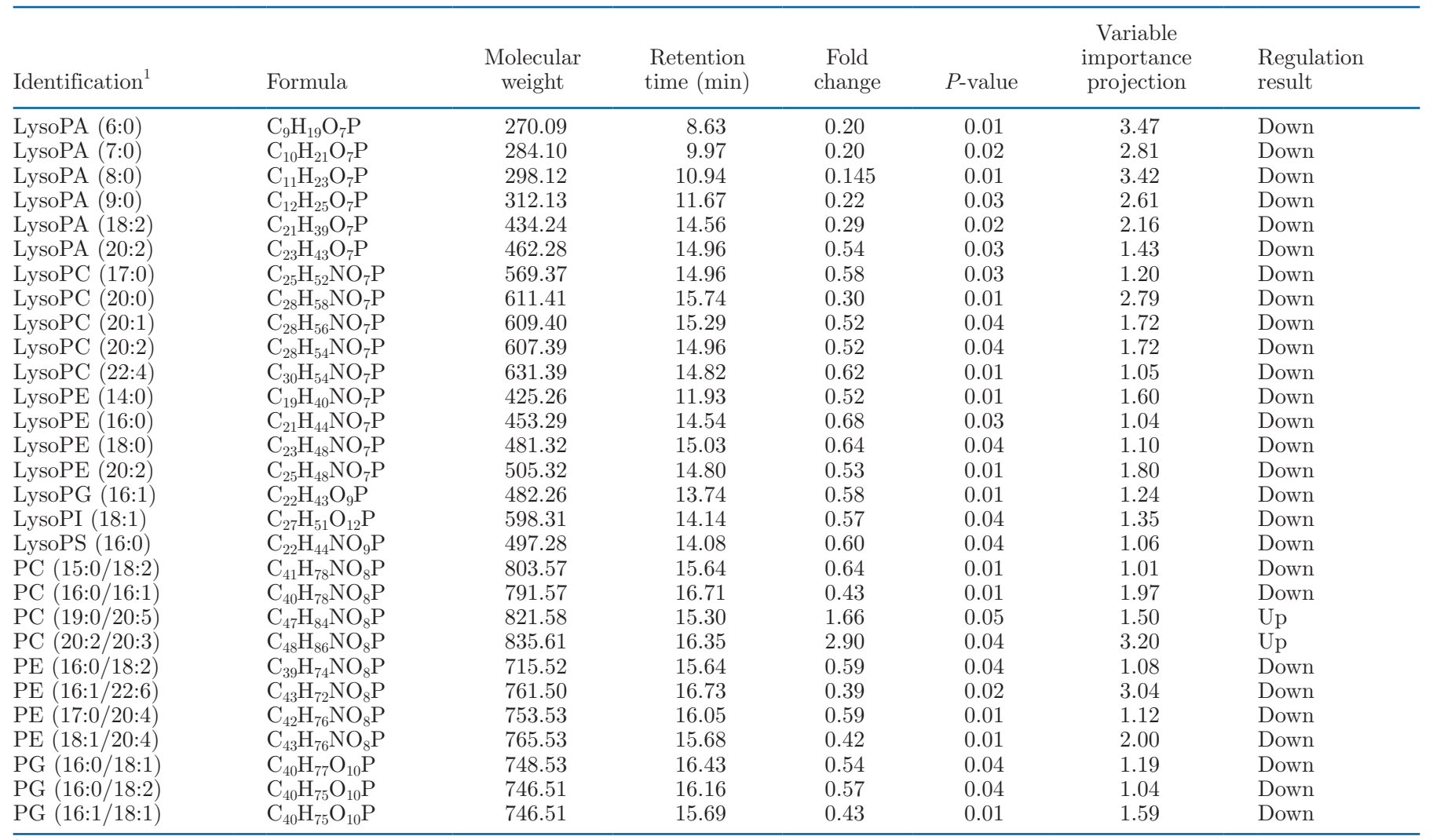

${ }^{1}$ LysoPA = lysophosphatidic acid; lysoPC = lysophosphatidylcholine; lysoPE = lysophosphatidylethanolamines; lysoPG = lysophosphatidylglycerols; lysoPI = lysophosphatidylinositol; lysoPS = lysophosphatidylserine; $\mathrm{PC}=$ phosphatidylcholine; $\mathrm{PG}=$ phosphatidylglycerol. 
metabolomic profiles of the groups revealed that MFEsweetened synbiotic yogurt can provide useful insights on the comprehensive understanding of the effects of different sweeteners. The results of this study revealed that high-fat diet and STZ injection induced metabolicrelated stress response, which resulted in dramatic alterations in T2DM. However, compared with the sucrose-containing yogurt, the MFE-sweetened synbiotic yogurt induced recovery from these alterations.

The liver has been associated with the pathogenesis of T2DM (Ko et al., 2015). Particularly, the develop- ment of T2DM is closely related to distinct changes in the profiles of liver lipids, including fatty acids and phospholipids, which are highly-rich lipid metabolites in the liver (Ha et al., 2012; Yore et al., 2014). There were significant differences $(P<0.05)$ between the metabolic profiles of the MYH and SIDR groups. In addition, the classification of lipid biomarkers revealed that compared with the SIDR group, a significant regulation of PC, PE, PG, lysoPA, lysoPC, lysoPE, lysoPG, lysoPI, and lysoPS was observed in the MYH group $(P<0.05)$. These lipid biomarkers are associated with

Table 2. Identified phospholipids biomarkers in the diabetic rats of the group treated with monk fruit extract-sweetened synbiotic yogurt ${ }^{1}$

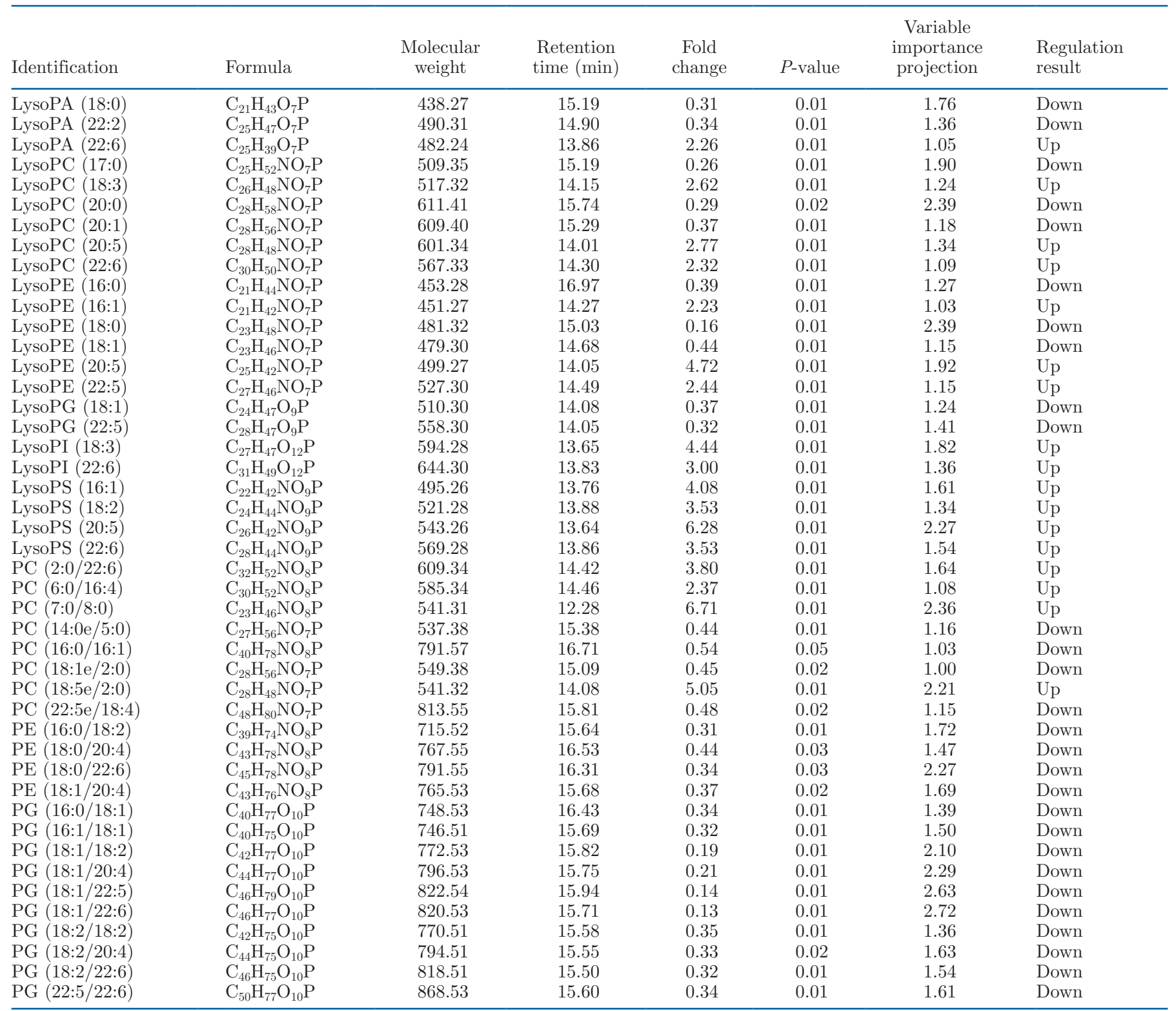

${ }^{1}$ LysoPA = lysophosphatidic acid; lysoPC = lysophosphatidylcholine; lysoPE = lysophosphatidylethanolamines; lysoPG = lysophosphatidylglycerols; lysoPI = lysophosphatidylinositol; lysoPS = lysophosphatidylserine; $\mathrm{PC}=$ phosphatidylcholine; $\mathrm{PG}=$ phosphatidylglycerol. 
Table 3. Identified fatty acid-hydroxy fatty acids (FAHFA) in the diabetic rats of the control yogurt (CY) group

\begin{tabular}{|c|c|c|c|c|c|c|c|}
\hline Identification & Formula & $\begin{array}{c}\text { Molecular } \\
\text { weight }\end{array}$ & $\begin{array}{l}\text { Retention } \\
\text { time (min) }\end{array}$ & $\begin{array}{l}\text { Fold } \\
\text { change }\end{array}$ & $P$-value & $\begin{array}{l}\text { Variable } \\
\text { importance } \\
\text { projection }\end{array}$ & $\begin{array}{l}\text { Regulation } \\
\text { result }\end{array}$ \\
\hline FAHFA $(20: 5 / 22: 3)$ & $\mathrm{C}_{42} \mathrm{H}_{66} \mathrm{O}_{4}$ & 634.50 & 13.93 & 2.35 & 0.01 & 1.83 & Up \\
\hline FAHFA $(22: 5 / 20: 2)$ & $\mathrm{C}_{42} \mathrm{H}_{68} \mathrm{O}_{4}$ & 636.51 & 14.36 & 2.44 & 0.01 & 1.92 & Up \\
\hline FAHFA $(18: 3 / 22: 3)$ & $\mathrm{C}_{40} \mathrm{H}_{66} \mathrm{O}_{4}$ & 610.50 & 13.95 & 1.62 & 0.01 & 1.05 & $\mathrm{Up}$ \\
\hline FAHFA $(20: 4 / 18: 1)$ & $\mathrm{C}_{38} \mathrm{H}_{64} \mathrm{O}_{4}$ & 584.48 & 14.18 & 1.68 & 0.01 & 1.16 & Up \\
\hline FAHFA $(18: 2 / 18: 1)$ & $\mathrm{C}_{36} \mathrm{H}_{64} \mathrm{O}_{4}$ & 560.48 & 14.22 & 1.61 & 0.01 & 1.08 & $\mathrm{Up}$ \\
\hline FAHFA $(22: 5 / 22: 4)$ & $\mathrm{C}_{44} \mathrm{H}_{68} \mathrm{O}_{4}$ & 330.26 & 14.35 & 2.78 & 0.01 & 2.22 & Up \\
\hline FAHFA $(20: 4 / 22: 4)$ & $\mathrm{C}_{42} \mathrm{H}_{66} \mathrm{O}_{4}$ & 634.50 & 14.29 & 2.67 & 0.01 & 2.01 & Up \\
\hline FAHFA $(14: 1 / 22: 3)$ & $\mathrm{C}_{36} \mathrm{H}_{62} \mathrm{O}_{4}$ & 558.47 & 13.50 & 1.73 & 0.02 & 1.13 & $\mathrm{Up}$ \\
\hline FAHFA $(16: 2 / 22: 3)$ & $\mathrm{C}_{38} \mathrm{H}_{64} \mathrm{O}_{4}$ & 584.48 & 13.75 & 2.27 & 0.02 & 1.67 & Up \\
\hline FAHFA $(20: 5 / 21: 1)$ & $\mathrm{C}_{41} \mathrm{H}_{68} \mathrm{O}_{4}$ & 624.51 & 13.94 & 1.76 & 0.04 & 1.37 & $\mathrm{Up}$ \\
\hline
\end{tabular}

diabetes and its metabolism (Ha et al., 2012; Kim et al., 2014; Yore et al., 2014). In addition, only 46 lipid biomarkers and 14 FAHFA biomarkers metabolites were detected in the CY group. This indicated that the different sweeteners had different effects on the lipid and FAHFA biomarkers of the diabetic rats. In addition, several lipid biomarkers, including PC, PE, lysoPC, lysoPE, lysoPS, and FAHFA, were enriched to a large extent. Kim et al. (2014) reported that highfat diet and STZ injection decreased the abundance of fatty acids and lipid biomarkers in the liver, which results in diabetes. The MFE-sweetened synbiotic yogurt effectively increased these indicators in the liver of the diabetic rats to a level close to the normal level (Table 2). A previous study reported that diabetes metabolic disorder can be improved by modulating lipid biomarkers (Kim et al., 2014). In this study, we verified that MFE-sweetened synbiotic yogurt can be used to prevent T2DM via the regulation of lipid and FAHFA biomarkers.

The MFE-sweetened synbiotic yogurt altered several hepatic metabolites related to metabolic diseases. In addition, changes in these metabolic profiles resulted in differences between the MYH and SIDR rats. To further explore and investigate the potential relationship between the metabolic pathways in the T2DM rats treated with MFE-sweetened synbiotic yogurt, a detailed pathway analysis was performed. Some of the targeted metabolites have been extensively investigated, whereas others have not. Based on the KEGG database and reference publication, a network diagraph was formed (Figure 1). The results revealed that trans-cinnamic acid and hydrocinnamic acid were the biomarkers in the phenylalanine metabolism pathway, indicating a variation in the metabolism in the rats. The trans-cinnamic acid was produced by Lphenylalanine, which is the trans-structure of cinnamic acid (Zang et al., 2015). Moreover, trans-cinnamic acid participated in numerous metabolic pathways, such as the biosynthesis of phenylpropanoid, ubiquinone, tryptophan metabolism, and other terpenoidquinone. In addition, Figure 1 suggests that trans-cinnamic acid and hydrocinnamic acid are downstream conciliators of phenylalanine, which play a significant role in the metabolism of phenylalanine. The MYH and SIDR groups exhibited significantly higher levels of hydrocinnamic acid and trans-cinnamic acid, indicating that the MFEsweetened synbiotic yogurt changed the phenylalanine

Table 4. Identified fatty acid-hydroxy fatty acids (FAHFA) in the diabetic rats of the group treated with yogurt containing monk fruit extract

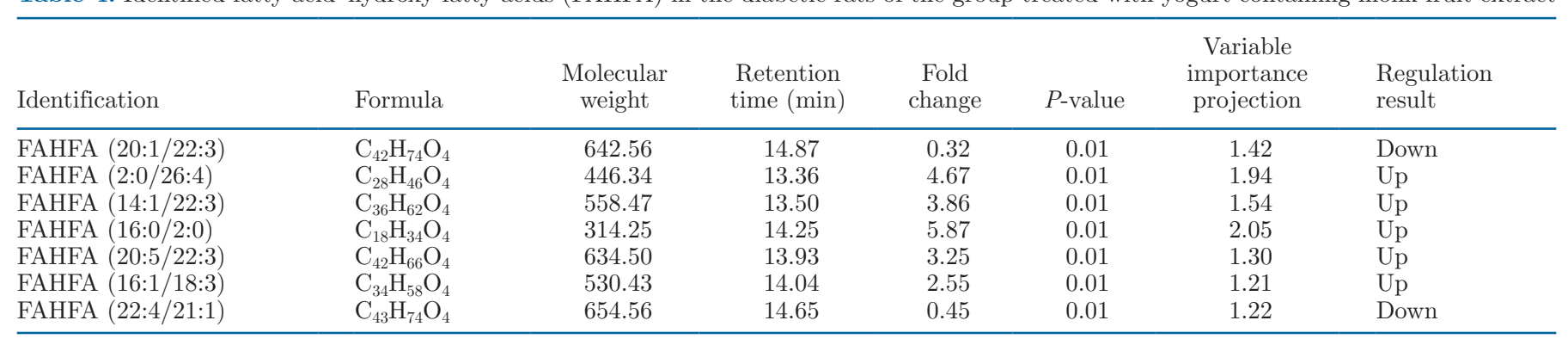




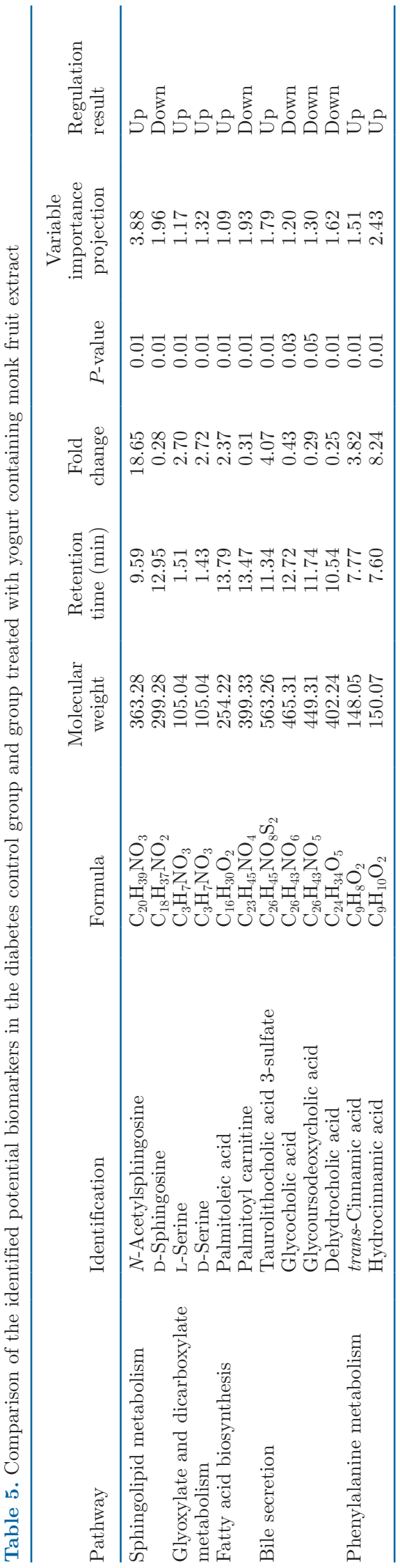

metabolism pathway of the T2DM rats in the MYH group.

D-Sphingosine and N-acetylsphingosine are middle metabolites in the metabolism of sphingolipid. The gavaging of the MFE-sweetened synbiotic yogurt increased the $\mathrm{N}$-acetylsphingosine level in the T2DM rats and decreased the D-sphingosine level. In mammals, D-sphingosine and its derivative (sphinganine) plays an important role in the formation of a signaling lipid (Roviezzo et al., 2016). Di Nardo et al. (2000) reported that $\mathrm{N}$-acetylsphingosine could restrain cell proliferation and induce apoptosis. A change in the metabolism of sphingolipid has been observed in diabetic patients, which may be related to the cell death mechanism (Fox et al., 2006). Researchers have reported the promising potential of sphingolipid metabolism as a potential metabolism pathway for treating T2DM.

Dehydrocholic acid, taurolithocholic acid 3-sulfate, and glycoursodeoxycholic acid are relevant for bile secretion. Figure 1 suggests that the 3 biomarkers are all downstream arbitrators of cholesterol, which are essential in bile secretion. Bile secretion is the major pathway for the catabolism of cholesterol to bile acids (Qi et al., 2015). Bile acids enable the regulation of cholesterol homeostasis, and can help in controlling glucose, lipid, and energy homeostasis (Li and Chiang, 2012). The gavaging of MFE-sweetened synbiotic yogurt increased the level of taurolithocholic acid 3-sulfate and decreased those of dehydrocholic acid and glycoursodeoxycholic acid. Accordingly, an enhancement in the bile secretion metabolism was observed in the T2DM rats of the MYH group.

Serine, L-serine, and D-serine are related to the metabolism of glyoxylate and dicarboxylate. Particularly, serine is an important intermediate in the metabolism of glyoxylate and dicarboxylate. D-Serine and L-serine are mutually involved in the conversion process in the metabolism of glyoxylate and dicarboxylate. L-Serine is categorized as an NEAA, and is relevant to the development of T2DM (Holm and Buschard, 2019). In this study, the gavaging of MFE-sweetened synbiotic yogurt increased the levels of L-serine, D-serine, and serine in the T2DM rats.

Currently, there are no comprehensive transcriptomic and metabolomic evaluation in T2DM research. This study identified numerous metabolic and transcriptional alterations induced by the oral administration of MFEsweetened synbiotic yogurt in T2DM rats. This study aimed to investigate the range of variations that should be anticipated in commonly identified metabolites and in transcripts when these "omics" are evaluated. Figure 3 indicates that the synthesis of acyl-CoA regulated the related pathway in the liver. Particularly, we found that the activation of 3 interconversion metabolic path- 


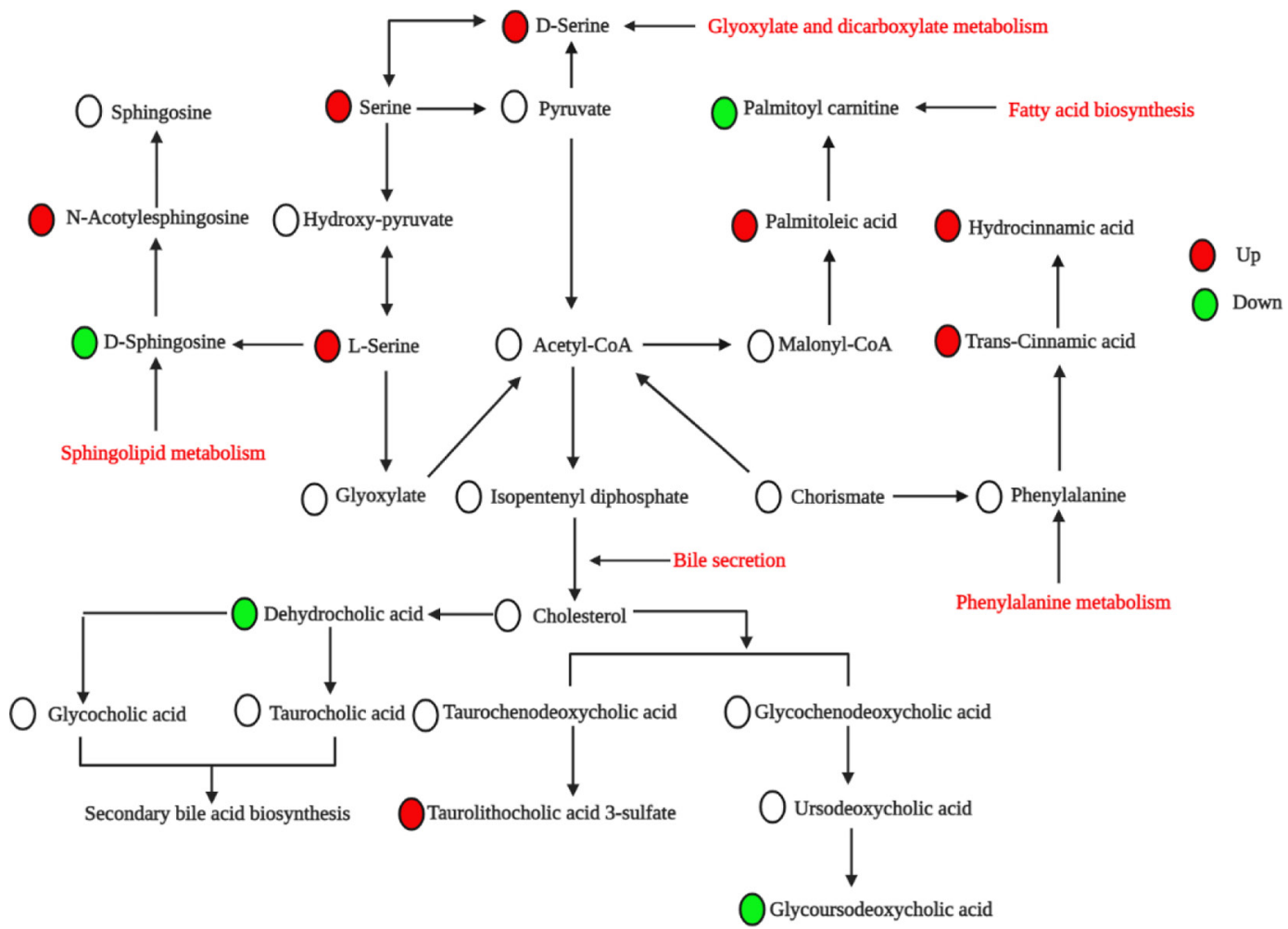

Figure 1. Correlation networks of the main potential biomarkers in response to the effects of monk fruit extract (MFE)-sweetened synbiotic yogurt (MYH) on the diabetic rats. The increased levels of metabolites by MYH are indicated in red; the decreased levels of metabolites by $\mathrm{MYH}$ are indicated in green.

ways (i.e., fatty acid biosynthesis, bile secretion, and glyoxylate and dicarboxylate metabolism) resulted in the gradual improvement of the metabolism disorders in T2DM rats by the MFE-sweetened synbiotic yogurt. Certain important transcripts affect the liver, owing to their roles in the metabolism of fatty acid biosynthesis.
The changes induced by these transcripts included concerted increases in mRNAs encoding key enzymes, such as Acaca (acetyl-CoA carboxylase $\alpha$ ), Acacb (acetylCoA carboxylase $\beta$ ), Acls5 (acyl-CoA synthetase 5), and Fasn (fatty acid synthase). In addition, the level of palmitoleic acid in the MYH group was higher than
A

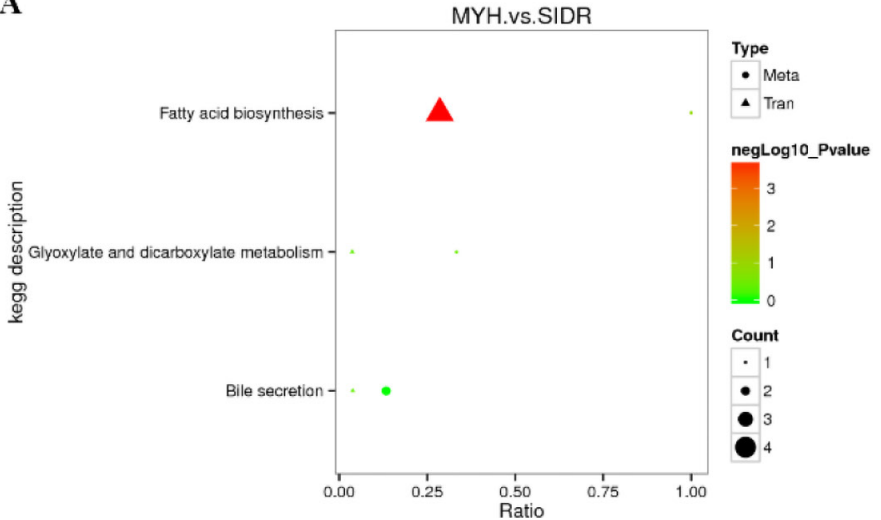

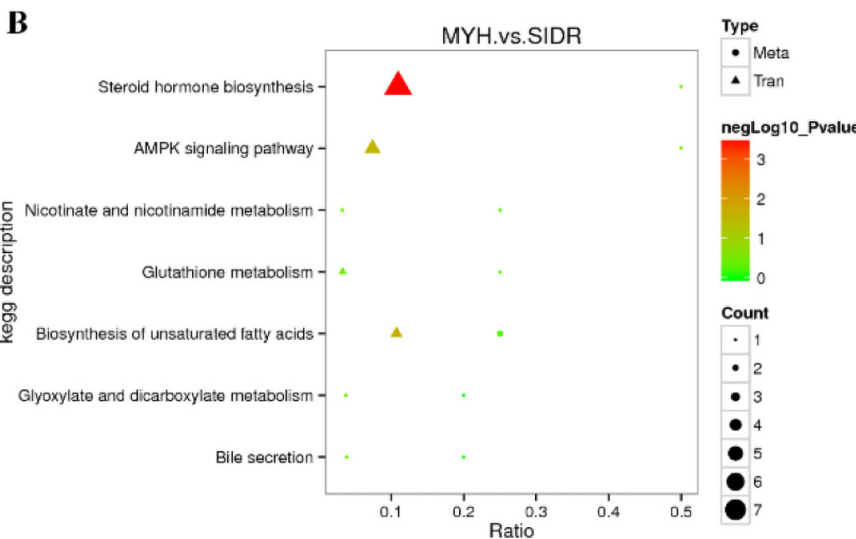

Figure 2. Transcriptomic and metabolomic evaluation for Kyoto Encyclopedia of Genes and Genomes (KEGG) pathway enrichment analysis of the livers of the rats in the monk fruit extract-sweetened synbiotic yogurt (MYH) and diabetes control (SIDR) groups. A: positive mode; B: negative mode. Tran $=$ transcriptomic; Meta $=$ metabolomic . 


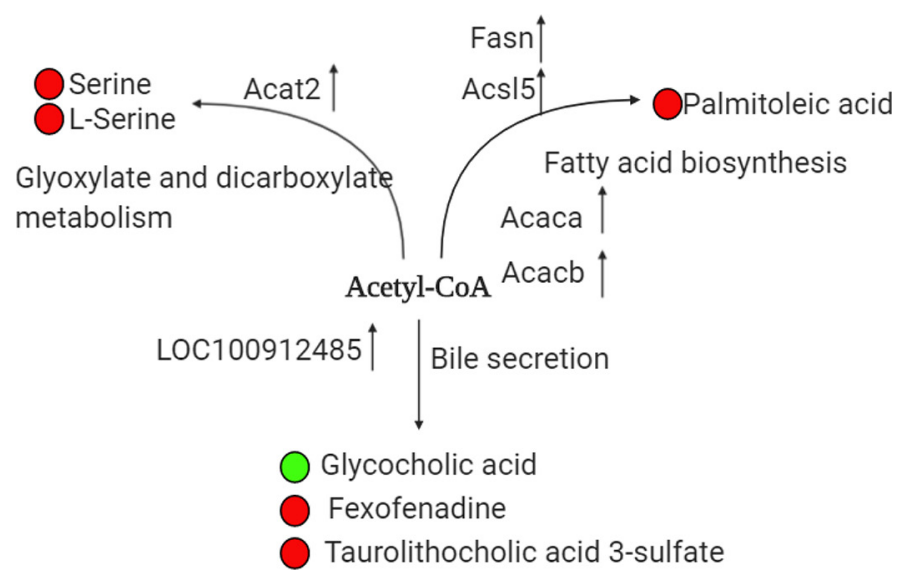

Figure 3. Ideogram illustration of the integrated gene expressions and metabolites in the livers of the diabetic rats in the monk fruit extract-sweetened synbiotic yogurt (MYH) group. Metabolites are indicated with names, and genes are indicated with symbols. The arrows indicate the regulations of the content or expression of the metabolites. The increased levels of metabolites by MYH are indicated in red, and the decreased levels of metabolites by MYH are indicated in green.

that in the SIDR group. Further, this study verified the ability of palmitoleic acid in preventing $\beta$-cell apoptosis induced by monosaccharides and fatty acids (Morgan and Dhayal, 2010), and found that palmitoleic acidrich foods can improve the characterization of lipid in body and animal model (Griel et al., 2008; Matthan et al., 2009). Further, the results of this study demonstrated that MFE-sweetened synbiotic yogurt improved the metabolic disorders in the T2DM rats by regulating their palmitoleic acid levels. In addition, MFE-sweetened synbiotic yogurt treatment increased the expression of hepatic Acat2 (acyl-CoA: cholesterol acyltransferase 2) and their downstream genes, which are involved in the metabolism of glyoxylate and dicarboxylate. The Acat2 gene significantly affected the level of serine in the liver $(P<0.05)$, which could be attributed to the enhancement in the metabolism of T2DM by serine (Holm and Buschard, 2019). In addition, acetyl-CoA regulated the upregulation of Sult2a6 (sulfotransferase family $2 \mathrm{~A}$, dehydroepiandrosterone preferring, member 6), which catalyzes the key step in bile secretion at the transcriptional level. The MFEsweetened synbiotic yogurt treatment increased the metabolism of the intermediates of bile acid (fexofenadine and taurolithocholic acid 3-sulfate) and decreased the metabolism of glycocholic acid, inferring the regulation of lipid metabolism in the T2DM rats via the increase in the levels of second bile acid. The results of this study indicated that the mechanisms involved in the mediated metabolic control of bile acid may be relevant in the pathogenesis of T2DM (Prawitt et al., 2011).

\section{CONCLUSIONS}

In this study, we found that MFE-sweetened synbiotic yogurt plays an active role in the prevention and progression of T2DM. In addition, the MFE-sweetened synbiotic yogurt improved the phospholipids and FAHFA profiles of the livers, indicating that they can be used as lipid biomarkers. Furthermore, MFE-sweetened synbiotic yogurt treatment improved the phenylalanine metabolism, sphingolipid metabolism, bile secretion, glyoxylate, and dicarboxylate metabolism in the T2DM rats. Furthermore, multiomics studies confirmed that the MFE-sweetened synbiotic yogurt regulated fatty acid biosynthesis, bile secretion, and glyoxylate and dicarboxylate metabolism, thus altering the levels of lipid biomarkers in the livers of the T2DM rats. The findings of this study will provide useful insights on the development of new strategies for exploring the function of MFE-sweetened synbiotic yogurt in T2DM rats. In addition, this study described the potential metabolism triggered by liver metabolite regulation.

\section{ACKNOWLEDGMENTS}

Financial support for this project was provided by a special grant from Northeast Agricultural University (Harbin, China). The authors have not stated any conflicts of interest.

\section{REFERENCES}

Ban, Q. 2022. T2DM metabolome data. Mendeley Data, V1. https:// doi.org/10.17632/6r9xwsbhx7.1.

Ban, Q., J. J. Cheng, X. M. Sun, Y. Q. Jiang, S. B. Zhao, X. Song, and M. R. Guo. 2020a. Effects of a synbiotic yogurt using monk fruit extract as sweetener on glucose regulation and gut microbiota in rats with type 2 diabetes mellitus. J. Dairy Sci. 103:2956-2968. https://doi.org/10.3168/jds.2019-17700.

Ban, Q., Z. H. Liu, C. W. Yu, X. M. Sun, Y. Q. Jiang, J. J. Cheng, and M. R. Guo. 2020b. Physiochemical, rheological, microstructural, and antioxidant properties of yogurt using monk fruit extract as a sweetener. J. Dairy Sci. 103:10006-10014. https://doi.org/10 .3168/jds.2020-18703.

Carrizo, D., O. P. Chevallier, J. V. Woodside, S. F. Brennan, M. M. Cantwell, G. Cuskelly, and C. T. Elliott. 2017. Untargeted metabolomic analysis of human serum samples associated with different levels of red meat consumption: A possible indicator of type 2 diabetes? Food Chem. 221:214-221. https://doi.org/10.1016/j .foodchem.2016.10.056.

Carter, P., L. J. Gray, J. Troughton, K. Khunti, and M. J. Davies. 2010. Fruit and vegetable intake and incidence of type 2 diabetes mellitus: Systematic review and meta-analysis. BMJ 341:c4229. https://doi.org/10.1136/bmj.c4229.

Di Nardo, A., L. Benassi, C. Magnoni, A. Cossarizza, S. Seidenari, and A. Giannetti. 2000. Ceramide 2 (N-acetyl sphingosine) is associated with reduction in Bcl-2 protein levels by Western blotting and with apoptosis in cultured human keratinocytes. Br. J. Dermatol. 143:491-497. https://doi.org/10.1111/j.1365-2133.2000.03700.x.

Đurašević, S., G. Nikolić, I. Zaletel, I. Grigorov, L. Memon, D. MitićĆulafić, P. Vujović, J. Đorđević, and Z. Todorović. 2020. Distinct 
effects of virgin coconut oil supplementation on the glucose and lipid homeostasis in non-diabetic and alloxan-induced diabetic rats. J. Funct. Foods 64:103601. https://doi.org/10.1016/j.jff.2019 .103601.

Fang, C., Q. Wang, X. Liu, and G. Xu. 2017. Metabolic profiling analysis of Siraitia grosvenorii revealed different characteristics of green fruit and saccharified yellow fruit. J. Pharm. Biomed. Anal. 145:158-168. https://doi.org/10.1016/j.jpba.2017.06.046.

Fox, T. E., X. Han, S. Kelly, A. H. Merrill Jr., R. E. Martin, R. E. Anderson, T. W. Gardner, and M. Kester. 2006. Diabetes alters sphingolipid metabolism in the retina: a potential mechanism of cell death in diabetic retinopathy. Diabetes 55:3573-3580. https:/ /doi.org/10.2337/db06-0539.

Gijsbers, L., E. L. Ding, V. S. Malik, J. De Goede, J. M. Geleijnse, and S. S. Soedamah-Muthu. 2016. Consumption of dairy foods and diabetes incidence: A dose-response meta-analysis of observational studies. Am. J. Clin. Nutr. 103:1111-1124. https://doi.org/ 10.3945/ajcn.115.123216.

Griel, A. E., Y. Cao, D. D. Bagshaw, A. M. Cifelli, B. Holub, and P. M. Kris-Etherton. 2008. A macadamia nut-rich diet reduces total and LDL-cholesterol in mildly hypercholesterolemic men and women. J. Nutr. 138:761-767. https://doi.org/10.1093/jn/138.4.761.

Ha, C. Y., J. Y. Kim, J. K. Paik, O. Y. Kim, Y. H. Paik, E. J. Lee, and J. H. Lee. 2012. The association of specific metabolites of lipid metabolism with markers of oxidative stress, inflammation and arterial stiffness in men with newly diagnosed type 2 diabetes. Clin. Endocrinol. (Oxf.) 76:674-682. https://doi.org/10.1111/ j.1365-2265.2011.04244.x.

Harada, N., M. Ishihara, H. Horiuchi, Y. Ito, H. Tabata, Y. A. Suzuki, Y. Nakano, R. Yamaji, and H. Inui. 2016. Mogrol derived from Siraitia grosvenorii mogrosides suppresses 3T3-L1 adipocyte differentiation by reducing cAMP-response element-binding protein phosphorylation and increasing AMP-activated protein kinase phosphorylation. PLoS One 11:e0162252. https://doi.org/10.1371/ journal.pone.0162252.

Holm, L. J., and K. Buschard. 2019. L-serine: A neglected amino acid with a potential therapeutic role in diabetes. Acta Pathol. Microbiol. Scand. Suppl. 127:655-659. https://doi.org/10.1111/apm .12987.

Howard, B. V., and J. Wylie-Rosett. 2002. Sugar and cardiovascular disease: A statement for healthcare professionals from the Committee on Nutrition of the Council on Nutrition, Physical Activity, and Metabolism of the American Heart Association. Circulation 106:523-527. https://doi.org/10.1161/01.CIR.0000019552.77778 .04 .

International Diabetes Federation. 2019. IDF Diabetes Atlas. Accessed Oct. 15, 2019. https://www.diabetesatlas.org.

Kim, H. Y., M. Kim, H. M. Park, J. Kim, E. J. Kim, C. H. Lee, and J. H. Y. Park. 2014. Lysophospholipid profile in serum and liver by high-fat diet and tumor induction in obesity-resistant BALB/c mice. Nutrition 30:1433-1441. https://doi.org/10.1016/j.nut.2014 .04 .013 .

Ko, S. H., M. K. Baeg, K. D. Han, S. H. Ko, and Y. B. Ahn. 2015. Increased liver markers are associated with higher risk of type 2 diabetes. World J. Gastroenterol. 21:7478. https://doi.org/10 .3748/wjg.v21.i24.7478.

Li, C., L. M. Lin, F. Sui, Z. M. Wang, H. R. Huo, L. Dai, and T. L. Jiang. 2014. Chemistry and pharmacology of Siraitia grosvenorii: A review. Chin. J. Nat. Med. 12:89-102. https://doi.org/10.1016/ S1875-5364(14)60015-7.

Li, T., and J. Y. Chiang. 2012. Bile acid signaling in liver metabolism and diseases. J. Lipids 2012:754067. https://doi.org/10.1155/ $2012 / 754067$.

Liu, H., X. Qi, K. Yu, A. Lu, K. Lin, J. Zhu, M. Zhang, and Z. Sun. 2019. AMPK activation is involved in hypoglycemic and hypolipidemic activities of mogroside-rich extract from Siraitia grosvenorii (Swingle) fruits on high-fat diet/streptozotocin-induced diabetic mice. Food Funct. 10:151-162. https://doi.org/10.1039/ $\mathrm{C} 8 \mathrm{FO} 01486 \mathrm{H}$.
Lu, J., G. Xie, W. Jia, and W. Jia. 2013. Metabolomics in human type 2 diabetes research. Front. Med. 7:4-13. https://doi.org/10.1007/ s11684-013-0248-4.

Matthan, N. R., A. Dillard, J. L. Lecker, B. Ip, and A. H. Lichtenstein. 2009. Effects of dietary palmitoleic acid on plasma lipoprotein profile and aortic cholesterol accumulation are similar to those of other unsaturated fatty acids in the F1B golden Syrian hamster. J. Nutr. 139:215-221. https://doi.org/10.3945/jn.108.099804.

Meierhofer, D., C. Weidner, and S. Sauer. 2014. Integrative analysis of transcriptomics, proteomics, and metabolomics data of white adipose and liver tissue of high-fat diet and rosiglitazone-treated insulin-resistant mice identified pathway alterations and molecular hubs. J. Proteome Res. 13:5592-5602. https://doi.org/10.1021/ pr5005828.

Mesnage, R., M. Biserni, S. Balu, C. Frainay, N. Poupin, F. Jourdan, E. Wozniak, T. Xenakis, C. A. Mein, and M. N. Antoniou. 2018. Integrated transcriptomics and metabolomics reveal signatures of lipid metabolism dysregulation in HepaRG liver cells exposed to PCB 126. Arch. Toxicol. 92:2533-2547. https://doi.org/10.1007/ s00204-018-2235-7.

Morgan, N. G., and S. Dhayal. 2010. Unsaturated fatty acids as cytoprotective agents in the pancreatic $\beta$-cell. Prostaglandins Leukot. Essent. Fatty Acids 82:231-236. https://doi.org/10.1016/j.plefa .2010.02.018.

Murphy, S. P., and R. K. Johnson. 2003. The scientific basis of recent US guidance on sugars intake. Am. J. Clin. Nutr. 78:827S-833S. https://doi.org/10.1093/ajcn/78.4.827S.

Pandey, K. R., S. R. Naik, and B. V. Vakil. 2015. Probiotics, prebiotics and synbiotics-a review. J. Food Sci. Technol. 52:7577-7587. https: //doi.org/10.1007/s13197-015-1921-1.

Pawar, R. S., A. J. Krynitsky, and J. I. Rader. 2013. Sweeteners from plants-with emphasis on Stevia rebaudiana (Bertoni) and Siraitia grosvenorii (Swingle). Anal. Bioanal. Chem. 405:4397-4407. https: //doi.org/10.1007/s00216-012-6693-0.

Prawitt, J., S. Caron, and B. Staels. 2011. Bile acid metabolism and the pathogenesis of type 2 diabetes. Curr. Diab. Rep. 11:160-166. https://doi.org/10.1007/s11892-011-0187-x.

Qi, Y., C. Jiang, J. Cheng, K. W. Krausz, T. Li, J. M. Ferrell, F. J. Gonzalez, and J. Y. L. Chiang. 2015. Bile acid signaling in lipid metabolism: Metabolomic and lipidomic analysis of lipid and bile acid markers linked to anti-obesity and anti-diabetes in mice. Biochim. Biophys. Acta. 1851:19-29. https://doi.org/10.1016/j.bbalip 2014.04.008.

Roviezzo, F., R. Sorrentino, V. M. Iacono, V. Brancaleone, M. Terlizzi, M. A. Riemma, A. Bertolino, A. Rossi, M. Matteis, G. Spaziano, A. Pinto, B. D'Agostino, and G. Cirino. 2016. Disodium cromoglycate inhibits asthma-like features induced by sphingosine-1-phosphate. Pharmacol. Res. 113:626-635. https://doi.org/10.1016/j .phrs.2016.09.014.

Sas, K. M., A. Karnovsky, G. Michailidis, and S. Pennathur. 2015. Metabolomics and diabetes: Analytical and computational approaches. Diabetes 64:718-732. https://doi.org/10.2337/db14-0509.

Schrezenmeir, J., and M. de Vrese. 2001. Probiotics, prebiotics, and synbiotics-Approaching a definition. Am. J. Clin. Nutr. 73:361S364S. https://doi.org/10.1093/ajcn/73.2.361s.

Su, H., T. Bao, L. Xie, Y. Xu, and W. Chen. 2020. Transcriptome profiling reveals the antihyperglycemic mechanism of pelargonidin-3-O-glucoside extracted from wild raspberry. J. Funct. Foods 64:103657. https://doi.org/10.1016/j.jff.2019.103657.

Uuh-Narváez, J. J., M. A. González-Tamayo, and M. R. Segura-Campos. 2021. A study on nutritional and functional study properties of Mayan plant foods as a new proposal for type 2 diabetes prevention. Food Chem. 341:128247. https://doi.org/10.1016/j.foodchem .2020.128247.

Vamanu, E., D. Pelinescu, F. Gatea, and I. Sârbu. 2019. Altered in vitro metabolomic response of the human microbiota to sweeteners. Genes (Basel) 10:535. https://doi.org/10.3390/genes10070535.

Wan, Z., S. Khubber, M. Dwivedi, and N. N. Misra. 2021. Strategies for lowering the added sugar in yogurts. Food Chem. 344:128573. 
Watson, R. R., and V. R. Preedy. 2019. Bioactive food as dietary interventions for diabetes. Page 1 in Food Chemistry. Academic Press.

Yore, M. M., I. Syed, P. M. Moraes-Vieira, T. J. Zhang, M. A. Herman, E. A. Homan, R. T. Patel, J. Lee, S. L. Chen, O. D. Peroni, A. S. Dhaneshwar, A. Hammarstedt, U. Smith, T. E. McGraw, A. Saghatelian, and B. B. Kahn. 2014. Discovery of a class of endogenous mammalian lipids with anti-diabetic and anti-inflammatory effects. Cell 159:318-332. https://doi.org/10.1016/j.cell.2014.09 .035 .

Zang, Y., T. Jiang, Y. Cong, Z. Zheng, and J. Ouyang. 2015. Molecular characterization of a recombinant Zea mays phenylalanine ammonia-lyase (ZmPAL2) and its application in trans-cinnamic acid production from L-phenylalanine. Appl. Biochem. Biotechnol. 176:924-937. https://doi.org/10.1007/s12010-015-1620-4.

Zhang, A., H. Sun, Y. Han, Y. Yuan, P. Wang, G. Song, X. Yuan, M. Zhang, N. Xie, and X. Wang. 2012. Exploratory urinary metabolic biomarkers and pathways using UPLC-Q-TOF-HDMS coupled with pattern recognition approach. Analyst 137:4200-4208. https: //doi.org/10.1039/c2an35780a.

Zhao, G., X. Hou, X. Li, M. Qu, C. Tong, and W. Li. 2018. Metabolomics analysis of alloxan-induced diabetes in mice using UPLC-QTOF-MS after Crassostrea gigas polysaccharide treatment. Int. J. Biol. Macromol. 108:550-557. https://doi.org/10.1016/j.ijbiomac .2017.12.057.

\section{ORCIDS}

Qingfeng Ban () https://orcid.org/0000-0001-7305-8447

Xiaomeng Sun ๑ https://orcid.org/0000-0002-1132-8584

Mingruo Guo @ https://orcid.org/0000-0002-7869-4618 